\title{
STRUCTURE AND INSTABILITIES OF AN IRRADIATED VISCOUS PROTOPLANETARY DISK
}

\author{
HIDEKO NOMURA \\ Department of Physics, Graduate School of Science, Kyoto University, Sakyo-ku, Kyoto 606-8502, \\ Japan; hnomura@tap.scphys.kyoto-u.ac.jp
}

\begin{abstract}
We investigate the structure and the stabilities of a protoplanetary disk, which is heated by viscous process in itself and by its central star. The disk is set to rotate with the Keplerian velocity and has the surface density distribution of the minimum mass solar nebula. We assume the vertical hydrostatic equilibrium and the radiative equilibrium at each point, and solve the two-dimensional radiative transfer equation by means of the Short Characteristic method in the spherical coordinate in order to determine the disk structure. Our calculation shows that at the outer region of the disk with a distance from the central star of $x>1$ AU the radiative heating from the inner disk dominates the viscous heating even near the midplane. It is because of the high temperature distribution in the optically thin surface layer and the relatively high disk height $\left(z_{\infty} \sim 0.7 x\right.$ at $\left.x \sim 1 \mathrm{AU}\right)$ as a consequence of the irradiation from the inner hot region of the disk. In addition, we examine the convective and the magnetorotational instabilities of the disk. As a result, the whole disk is convectively stable since the dusty region is not heated by the viscous dissipation from the midplane but by the radial radiative heating. On the other hand, almost all the disk is magnetorotationally unstable except for the region near the equatorial plane of $2 \mathrm{AU}<x<10 \mathrm{AU}$. Finally we discuss the growth and the size distribution of dust particles in the disk, which suggests that there exist $\mathrm{cm}$-sized particles in the surface layer, namely, in the exposed region of the disk.
\end{abstract}

Subject headings: accretion, accretion disks — circumstellar matter — instabilities planetary systems: protoplanetary disks — radiative transfer

\section{INTRODUCTION}

It has been established that there exist circumstellar disks around a large fraction of young stellar objects (YSOs), some of which will evolve to form planetary systems analogous to our Solar System (Wilking, Lada, \& Young 1989; Stauffer et al. 1994; Haisch, Lada \& Lada 2001). Infrared excesses over the photospheric emissions, often observed in the spectral energy distributions (SEDs) of YSOs, are considered to be radiated from dusty circumstellar disks (Cohen 1974; Adams \& Shu 
1985; Kenyon \& Hartmann 1987; Beckwith \& Sargent 1993; Hartmann 1998). Furthermore, the disk-structure images with radii of $\sim 100 \mathrm{AU}$ are sometimes observed around YSOs at optical and near-infrared wavelengths as silhouettes against the background bright nebula (O'Dell et al. 1993; O'Dell 1998; Bally, O'Dell \& McCaurean 2000) or as optically thick dust lanes with optically thin reflection nebulae, absorbing and scattering light from their central stars (Burrows et al. 1996; Padgett et al. 1999).

These circumstellar disks play vital roles on the star and planetary system formation: for example, they transform angular momentum outward and accrete mass to their central stars (e.g., Spitzer 1978; Shu, Adams, \& Lizano 1987; Bodenheimer 1995; Papaloizou \& Lin 1995; Nomura \& Mineshige 2000; Stone et al. 2000), and they form planets in them through the various processes of dust grain growth (e.g., Safronov 1969; Weidenschilling \& Cuzzi 1993; Beckwith, Henning \& Nakagawa 2000). The turbulence in the disks is one of the keys to elucidate their structures and evolutions. The turbulent viscosity in differentially rotating disks transforms the angular momentum and mass, and releases the gravitational energy of accreted mass as heat through the viscous dissipation (e.g., Shakura \& Sunyaev 1973; Lynden-Bell \& Pringle 1974; Pringle 1981). A number of mechanisms to excite turbulent motion have been investigated for various regions of disks in varied evolutional stages, such as the thermal convection, the magnetorotational instability, the shear motion due to differential rotation, the infalling envelope, the stellar wind, and so forth (e.g., Lin \& Papaloizou 1980; Cabot et al. 1987; Balbus \& Hawley 1991; Sano et al. 2000; Zel'dovich 1981; Sekiya 2000; Elmegreen 1978; Cameron 1978), but we do not yet have a universally accepted scenario. Meanwhile, many studies have been done on the structure of such optically thick viscous (active) protostellar disk under the assumptions of the vertical hydrostatic equilibrium and the energy balance between the viscous heating and the radiative cooling, sometimes in the context of explaining the FU Ori outburst phenomena (e.g., Lin \& Papaloizou 1980; Pringle 1981; Lin \& Papaloizou 1985; Kawazoe \& Mineshige 1993; Bell \& Lin 1993).

In order to reproduce the structure of protoplanetary disk, however, it is not sufficient to evaluate only the vertical viscous heating from the midplane since the disks are irradiated from the central star and the inner hot region of the disk (e.g., Kenyon \& Hartmann 1987; Chiang \& Goldreich 1997; D'Alessio et al. 1998; Bell 1999). This irradiation process is noteworthy since it heats up and flares up the disk surface, reproducing the observed flat SEDs of far-infrared radiation from YSOs (the radiation flux of $\nu F_{\nu} \propto \nu^{0}$ ), which is not recreated by radiation from geometrically thin accretion disks (e.g., Lynden-Bell \& Pringle 1974; Adams, Lada, \& Shu 1987). The flared features of optically thick disks are in fact observed as shadows against scattering starlight in optically thin circumstellar matter (e.g., Burrows et al. 1996; Padgett et al. 1999). The profiles of such passive disks irradiated only from their central stars have been investigated under the assumption of the vertical hydrostatic equilibrium and with a simple model of radiative transfer of starlight, without and with the turbulent viscous heating (e.g., Kenyon \& Hartmann 1987; Chiang \& Goldreich 1997; Hubeny 1991; D'Alessio et al. 1998).

In this paper we strictly treat the radiation processes by solving the two-dimensional radiative 
transfer equation so as to investigate the structure and the instabilities of an axisymmetric disk under the influences of the viscous heating and the reprocessing of radiation from the central star and the inner hot region of the disk. In the next section we present the disk model, which is heated by the viscous dissipation and its central star, and assumed to satisfy the vertical hydrostatic equilibrium and the local radiative equilibrium. In $\S 3$ we calculate the density and temperature distributions of the disk. The convective and the magnetorotational instabilities of the disk, which will excite turbulent motion, are investigated in $\S 4$, and the dust size distribution in the disk is discussed by means of the turbulent-eddy-trapping model for dust growth in $\S 5$. Finally we summarize the results in $\S 6$.

\section{DISK MODEL}

We consider an axisymmetric disk surrounding a central star with physical parameters of the typical T Tauri stars; the mass of $M_{*}=0.5 M_{\odot}$, the radius of $R_{*}=2 R_{\odot}$, and the temperature of $T_{*}=4000 \mathrm{~K}$ (e.g., Kenyon \& Hartmann 1995). We investigate the density and temperature distributions of the disk under the assumptions of the hydrostatic equilibrium in the vertical direction $(\S 2.1)$ and the radiative equilibrium at each point $(\S 2.3)$. We also hypothesize that dust and gas have the same temperature distribution allover the disk in this paper. For a matter of convenience, we adopt the cylindrical coordinate $(x, z)$ in $\S 2.1$ and the spherical coordinate $(r, \Theta)$ [where $r \equiv\left(x^{2}+z^{2}\right)^{1 / 2}$ and $\left.\Theta \equiv \tan ^{-1}(x / z)\right]$ in $\S 2.3$, in both of which we put the central star at the origin.

\subsection{Hydrostatic Equilibrium in the Vertical Direction}

In order to determine the density distribution, $\rho(x, z)$, we assume the vertical hydrostatic equilibrium,

$$
\frac{d P}{d z}=\frac{c_{\mathrm{s}}^{2}}{\rho} \frac{d \rho}{d z}=-\rho g_{z}
$$

The sound speed $c_{\mathrm{s}}$ is defined as $c_{\mathrm{s}}{ }^{2} \equiv d P / d \rho=k T / m_{\mu}$, where $P, T, k$ and $m_{\mu}$ represent the pressure, the temperature, the Boltzmann's constant, and the mean molecular mass, respectively, and we adopt $m_{\mu}=2.3 m_{\mathrm{H}}\left(m_{\mathrm{H}}\right.$ is the hydrogen mass). The circumstellar disks around T Tauri stars are considered to be rotationally supported. Then the gravitational force in the vertical direction $g_{z}$ is written as $g_{z}=\Omega^{2} z$ with the Kepler frequency,

$$
\Omega(x)=\left(G M_{*} / x^{3}\right)^{1 / 2} \approx 1.4 \times 10^{-7} \mathrm{~s}^{-1}(x / 1 \mathrm{AU})^{-3 / 2},
$$

where we adopt $M_{*}=0.5 M_{\odot}$ for the mass of the central star. For the boundary condition, we put $\rho\left(x, z_{\infty}\right) \equiv 3.8 \times 10^{-22} \mathrm{~g} \mathrm{~cm}^{-3}$, which corresponds to the typical number density of molecular clouds, $n \approx 10^{2} \mathrm{~cm}^{-3}$ (where the mean molecular mass of $m_{\mu}=2.3 m_{\mathrm{H}}$ is assumed). The height of 
the disk $z_{\infty}$ is determined by the condition,

$$
\int_{-z_{\infty}}^{z_{\infty}} \rho(x, z) d z=\Sigma(x)
$$

where the surface density of the disk $\Sigma$ is put to agree with that of the minimum mass solar nebula (e.g., Hayashi, Nakazawa, \& Nakagawa 1985),

$$
\Sigma(x)=1.7 \times 10^{3} \mathrm{~g} \mathrm{~cm}^{-2}(x / 1 \mathrm{AU})^{-3 / 2} .
$$

Solving equation (1) with the above conditions and the temperature distribution $T(x, z)$, which is derived in subsection $\S 2.3$, by means of the fourth-order Runge-Kutta method, we get the density distribution $\rho(x, z)$.

\subsection{Heating Sources}

For the heating sources of the disk we consider two kinds of processes; the viscous dissipation in itself and the gravitational energy release associated with the contraction of its central T Tauri star. In protoplanetary disks it is believed that some kinds of instabilities (see $\S 4$ ) cause the turbulent motion and then induce the angular momentum and mass transfer. This process is accompanied with the heating of the disks via viscous dissipation of turbulence. Following the so-called $\alpha$-viscous model, we represent it by putting heating source at the midplane of the disk (at $z=0, \Theta=\pi / 2$ ) with a heating rate of

$$
Q_{\mathrm{vis}}^{+}=(9 / 4) \Sigma \alpha c_{\mathrm{s} 0}^{2} \Omega,
$$

where $c_{\mathrm{s} 0}$ is the sound speed at the midplane. In this model the kinetic viscosity is prescribed as $\nu_{\mathrm{vis}}=\alpha c_{\mathrm{s} 0} H=\alpha c_{\mathrm{s} 0}^{2} / \Omega$, where $H$ is the scale height of the disk (e.g., Shakura \& Sunyaev 1973; Pringle 1981; Kato, Fukue \& Mineshige 1998), and $\alpha=10^{-2}$ is adopted here. The vicinity of the midplane at the inner region of the disk is mainly heated by diffusive radiation from this viscous heating source as we will see in $\S 3$. Besides, we put thermal radiation source at the stellar surface (at $r=R_{*}$ ) with a heating rate of

$$
Q_{\mathrm{star}}^{+}=\sigma T_{*}^{4},
$$

where $\sigma$ is the Stefine-Boltzmann constant, and examine the reprocessing of radiation from the central star in the disk by solving the two-dimensional radiative transfer equation as we describe in the following subsection $(\S 2.3)$ and Appendix. For the stellar temperature $T_{*}=4000 \mathrm{~K}$ is adopted. We here comment that the irradiation not only from the central star but also from the inner hot disk on the surface region of the outer disk is automatically simulated in our calculation, and the latter dominates the former in our model as we will see in $\S 3$. 


\subsection{Radiative Equilibrium}

Evaluating the temperature distribution $T(r, \Theta)$, we assume the radiative equilibrium (that is, the emitted and absorbed radiation are balanced) at each point $(r, \Theta)\left[r \equiv\left(x^{2}+z^{2}\right)^{1 / 2}\right.$ is the distance from a central star and $\Theta \equiv \tan ^{-1}(x / z)$ is the angle from the $z$-axis] as

$$
4 \pi \int_{0}^{\infty} \kappa_{\nu}(r, \Theta) B_{\nu}[T(r, \Theta)] d \nu=\int_{0}^{\infty} \kappa_{\nu}(r, \Theta) J_{\nu}(r, \Theta) d \nu
$$

where $\nu$ is the photon frequency, $B_{\nu}(T)$ the Plank function for blackbody radiation, and $\kappa_{\nu}$ the monochromatic opacity given by the model in the following subsection (§2.4). In equation (7) local thermodynamics equilibrium $\eta_{\nu}(r, \Theta)=\kappa_{\nu}(r, \Theta) B_{\nu}[T(r, \Theta)]$ is assumed, where $\eta_{\nu}$ is the monochromatic emissivity (e.g., Mihalas 1978). The mean intensity $J_{\nu}(r, \Theta)$ is given by integrating the specific intensity $I_{\nu}(r, \Theta ; \mu, \phi)$ of radiation field for all solid angles $d \omega \equiv d \mu d \phi$ as

$$
J_{\nu}(r, \Theta)=\frac{1}{4 \pi} \int_{0}^{2 \pi} \int_{-1}^{1} I_{\nu}(r, \Theta ; \mu, \phi) d \mu d \phi,
$$

where $\mu$ is the cosine of the angle between the ray direction and the vector $\overrightarrow{O P}(O=[0,0], P=$ $[r, \Theta])$, and $\phi$ is the angle between the projection of the ray to the plane whose normal is $O P$ and the line $(\phi=0)$ on the plane parallel to the midplane of the disk $(z=0, \Theta=\pi / 2)$ (cf. Fig. 3 of Dullemond \& Troulla 2000). We here divide the total specific intensity $I_{\nu}$ into three parts as

$$
I_{\nu}=I_{\nu}^{*}+I_{\nu}^{\mathrm{vis}}+I_{\nu}^{\mathrm{th}}
$$

where $I_{\nu}^{*}$ and $I_{\nu}^{\mathrm{vis}}$ represent radiation directly from the heating sources described in $\S 2.2$, the central star and the viscous dissipation at the midplane of the disk, respectively, and $I_{\nu}^{\text {th }}$ arises from thermal emission of dust grains in the disk (e.g., Shu 1991; Efstathiou \& Rowan-Robinson 1990). We note that we neglect the radiative scattering for the simplicity in this paper. Each of them is derived from radiative transfer equation,

$$
\frac{d I_{\nu}^{s}}{d s}=\rho \kappa_{\nu}\left(S_{\nu}^{s}-I_{\nu}^{s}\right)
$$

where the source functions $S_{\nu}^{*}=S_{\nu}^{\mathrm{vis}}=0$ and $S^{\text {th }}=B_{\nu}$. From the above equation, the intensity $I_{\nu}^{*}$ and $I_{\nu}^{\mathrm{vis}}$ are derived with $T_{*}=T\left(r=R_{*}, \Theta\right)$ and $T_{\mathrm{vis}}(x)=T(r, \Theta=\pi / 2)$ as

$$
I_{\nu}^{*}(r, \Theta ; \mu, \phi)=B_{\nu}\left(T_{*}\right) e^{-\tau_{\nu}(r, \Theta)}
$$

for $\mu<-\left(r^{2}-R_{*}^{2}\right)^{1 / 2} / r$ [that is, $(\mu, \phi)$ originates from the central star] and $I_{\nu}^{*}(r, \Theta ; \mu, \phi)=0$ otherwise, and

$$
I_{\nu}^{\mathrm{vis}}(r, \Theta ; \mu, \phi)=B_{\nu}\left[T_{\mathrm{vis}}(r)\right] e^{-\tau_{\nu}(r, \Theta)}
$$

when $(\mu, \phi)$ originates form the midplane $(r, \Theta=\pi / 2)=(x, z=0)$ and $I_{\nu}^{\mathrm{vis}}(r, \Theta ; \mu, \phi)=0$ otherwise. The viscous heating temperature $T_{\text {vis }}(r)$ is related with $c_{\mathrm{s} 0}$ in equation (5) as $c_{\mathrm{s} 0}^{2}=k T_{\mathrm{vis}} / m_{\mu}$. The intensity $I_{\nu}^{\text {th }}$ is derived by integrating equation (10) along a ray, $s$ (which is directed to $[\mu, \phi]$ ), as

$$
I_{\nu}^{\mathrm{th}}(r, \Theta ; \mu, \phi)=\int_{0}^{s} \kappa_{\nu}\left(r^{\prime}, \Theta^{\prime}\right) \rho\left(r^{\prime}, \Theta^{\prime}\right) B_{\nu}\left[T\left(r^{\prime}, \Theta^{\prime}\right)\right] e^{-\tau_{\nu}\left(r^{\prime}, \Theta^{\prime}\right)} d s^{\prime} .
$$


In equations (11)-(13) $\tau_{\nu}(r, \Theta)$ represents the specific optical depth at the point $(r, \Theta)$ from the radiation source $\left(r^{\prime}, \Theta^{\prime}\right)$, given by

$$
\tau_{\nu}(r, \Theta)=\int_{0}^{s} \kappa_{\nu}\left(r^{\prime}, \Theta^{\prime}\right) \rho\left(r^{\prime}, \Theta^{\prime}\right) d s^{\prime}
$$

Together with the density distribution $\rho(r, \Theta)$ obtained in $\S 2.1$, we solve equations (7)-(14) selfconsistently to get the temperature distribution $T(r, \Theta)$ in the disk. The numerical method to solve equation (7) is described in Appendix A.1, and that to calculate equations (11)-(14) is in A.2. We note that we do not consider energy transport by convection here, but it turns out in $\S 4.1$ that this is permissible since the whole of the disk is in fact convectively stable in our disk model.

\subsection{Opacity}

In this paper we consider that the disk consists of dusty medium (in which dust is well mixed with gas) at low temperature $(T<2300 \mathrm{~K})$ and only of gaseous matter at $T>2300 \mathrm{~K}$. For dusty medium we use the frequency dependent absorption coefficient $\kappa_{\nu}$ of Adams \& Shu (1985, 1986; see also Draine \& Lee 1984). In their model the dust is constituted by three types of components of graphite and olivine silicate grains, and water icy mantle which coats the grains. They are assumed to evaporate at the characteristic dust destruction temperatures of $2300 \mathrm{~K}$ (graphite), $1500 \mathrm{~K}$ (silicate), and $150 \mathrm{~K}$ (ice). For gaseous matter we provisionally take the weighted transmission averaged opacity, the Rosseland mean opacity $\kappa_{\mathrm{R}}$, defined as $1 / \kappa_{\mathrm{R}} \equiv \int_{0}^{\infty}\left(1 / \kappa_{\nu}\right)\left(\partial B_{\nu} / \partial T\right) d \nu / \int_{0}^{\infty}\left(\partial B_{\nu} / \partial T\right) d \nu$. The density and temperature dependent model of Bell \& Lin (1994), $\kappa_{\mathrm{R}}=\kappa_{i} \rho^{a} T^{b}$, is adopted here, where $\kappa_{i}, a$ and $b$ are given for each of eight states of gas. It represents the Alexander/Cox/Stewart opacity and covers the wide temperature range of $10^{3} \mathrm{~K}<T<10^{7} \mathrm{~K}$ (see Bell \& Lin 1994 in details and see also Lin \& Papaloizou 1985). The applicability of this approximate treatment of the opacity for gas is discussed in $\S 3$.

\section{DENSITY AND TEMPERATURE PROFILES OF THE DISK}

Solving the equations of the vertical hydrostatic equilibrium (1) and the local radiative equilibrium (7) iteratively together with the two-dimensional radiative transfer equation (10) as we mentioned in the previous section and Appendix, we obtain the self-consistent density and temperature distributions of the disk heated by the viscous process in itself and by its central star. Figure 1a shows the contour plots of the resultant temperature (solid lines) and density (dotted lines) profiles in the $x-z$ plane of the 'irradiated disk' [which means that it is the result of the two-dimensional calculation of radiative transfer]. The contour levels are $T=10,10^{2}, 10^{3}, 10^{4}$, and $10^{5} \mathrm{~K}$ for the temperature, and $\rho=10^{-18}, 10^{-16}, 10^{-14}, 10^{-12}, 10^{-10}$, and $10^{-8} \mathrm{~g} \mathrm{~cm}^{-3}$ for the density. We also plot the vertical temperature and density distributions at $x=0.046,0.1,0.21,0.46,1.0,2.1,4.6,10$, and $21 \mathrm{AU}$ in Figure 2a and 3a, respectively. We here mention the applicability of our opacity 
model for gas $(T>2300 \mathrm{~K})$. Whereas we use the Rosseland mean opacity in this paper as we stated in $\S 2.4$, the Planck mean opacity is to be used in equation (13) and the left side of equation (7), and the energy-weighted one in the right side of equation (7) (see Hubeny 1990). This discrepant treatment of the opacity will modify the temperature profile at the optically thin region of the inner disk because the difference among these opacities goes up to several orders of magnitude at the maximum (e.g., Alexander \& Ferguson 1994). The temperature near the midplane is, however, hardly affected since it is determined by equation (A1) as we stated in Appendix. For comparison we display those of the 'non-irradiated disk' [which is derived from the energy transport equation only in the vertical direction (A1)] in Figure 1b, 2b, and $3 \mathrm{~b}$ in the same manner as Figure 1a-3a. The constant temperature near the surface in Figure $1 \mathrm{~b}$ and $2 \mathrm{~b}$ is due to our inappropriate use of the diffusion approximation (eq. [A1]) even at the optically thin region.

The remarkable differences between the 'irradiated' and 'non-irradiated' cases appear in the rises of temperature at the surface region and at the equatorial plane outside $x \sim 1 \mathrm{AU}$ of the 'irradiated disk'. As a result, the vertical height of the 'irradiated disk' becomes higher than that of the 'non-irradiated disk'. The effect of this flaring is due to the irradiation from the inner region and conspicuous at the outer disk, as is modeled in Kenyon \& Hartmann (1987), especially at the optically thin surface layer (Chiang \& Goldreich 1997; D'Alessio et al. 1998). The main source of the irradiation is, however, the inner hot disk rather than the central star in this case, contrasting with the previous works. We next discuss the reason for the radiative heating from the inner disk to dominate the viscous heating at $x>1 \mathrm{AU}$. Now the energy density due to the viscous heating at the midplane of the disk decreases with radius approximately as $E_{\mathrm{vis}}=a T_{\mathrm{vis}}^{4} \propto r^{-10}$, which is derived from the equations of the energy balance and the radiative transfer in the vertical direction, $(9 / 4) \Sigma \alpha c_{\mathrm{s} 0}^{2} \Omega=\sigma T_{\text {eff }}^{4} \sim(4 / 3) a c T_{\text {vis }}^{4} / \kappa_{\mathrm{R}} \Sigma$, where we use $\Sigma \propto r^{-3 / 2}$, $\Omega \propto r^{-3 / 2}, c_{\mathrm{s} 0}^{2} \propto T_{\text {vis }}$, and $\kappa_{\mathrm{R}} \propto T_{\text {vis }}^{6 / 5}$ (dust grains mainly contribute to the opacity in this region). Next let us consider the radial dependence of the energy density due to the radiative heating from the inner disk. If we postulate the extreme case that the height was large enough to adopt the plane-parallel approximation in the radial direction, the dependence would became $E_{\text {rad }}=a T_{\text {rad }}^{4} \sim\left(3 F_{\text {rad }} / 4 c\right) \int \kappa_{\mathrm{R}} \rho d r \propto r^{-5}$, where $F_{\text {rad }}$ (= const.) is the radial radiative flux and we use $\kappa_{\mathrm{R}} \propto T_{\text {rad }}^{6 / 5}$ and $\rho \propto r^{-5 / 2}$ from our calculation. In this case the radiative heating would surely dominate the viscous heating. On the contrary, if the disk is geometrically thin enough, the radiative heating from the inner disk becomes negligible. Thus one of the reasons for our result is the high vertical height of the disk. In addition the temperature at the surface layer is higher than that at the equatorial plane, which allows the effect of radiative heating from the inner region stronger.

In conclusion our results show that the surface layer of the disk is heated up and flared via the irradiation from the inner hot disk, and that the radiative heating from the inner disk dominates the viscous heating at $x>1 \mathrm{AU}$ because of the high disk height and the high temperature at the disk surface in this case. 


\section{DISK INSTABILITIES}

In protoplanetary disks it is believed that some kinds of instabilities cause the turbulent motion, and then, induce the angular momentum and mass transfer, and/or the energy transport. A number of processes have been proposed, among which we examine in this section two of intrinsical mechanisms in the disks before the dust settling stage: the convective instability $(\S 4.1)$ and the magnetorotational instability ( $\$ 4.2)$, making use of the temperature and density profiles obtained in the previous section.

\subsection{Convective Instability}

It has been suggested that the protoplanetary disks are convectively unstable because they consist of dusty components whose opacity has a temperature dependence of $\kappa_{\mathrm{R}} \propto T^{\beta}, \beta \geq 1$, and then causes a superadiabatic gradient against the direction of gravitational force (Lin \& Papaloizou 1980, see also Cameron \& Pine 1973; Ruden, Papaloizou, \& Lin 1988; Kley, Papaloizou, \& Lin 1993; Stone \& Balbus 1996). Now we examine the convective instability of the disk, which has the temperature and density distributions shown in $\S 3$, using the criterion of

$$
\Delta_{z}-\Delta_{\mathrm{ad}}>0, \quad \Delta_{z} \equiv \frac{\partial \log T}{\partial \log P}, \quad \Delta_{\mathrm{ad}} \equiv \frac{\gamma-1}{\gamma},
$$

where $\gamma$ is the specific heat ratio and the pressure satisfies $P=\gamma c_{\mathrm{s}}^{2} \rho$ (e.g., Cox 1980). We here take $\gamma=7 / 5$ since the main component of the disk is molecular hydrogen. As a result, we find that the whole of the 'irradiated disk' [obtained from the two-dimensional calculation of radiative transfer] is convectively stable, while the dusty region $(T<2300 \mathrm{~K})$ of the 'non-irradiated disk' [derived from only the vertical radiative transfer] is unstable (Fig. 4b, dotted stripe region). The unstable region disappears in the 'irradiated disk' because its dusty region is not heated from the midplane but from the inner region as we see in $\S 3$.

\subsection{Magnetorotational Instability}

Magnetorotational instability is one of the promising turbulent sources in accretion disks (Velhikov 1959; Balbus \& Hawley 1991; Balbus \& Hawley 1998 and references therein). When a differentially rotating, fully ionized, magnetized disk satisfies the condition,

$$
2 \pi v_{\mathrm{A}} / \Omega \leq H
$$

the disk is magnetorotationally unstable, where $\Omega$ and $H$ are the rotational frequency and the disk scale height, respectively, and $v_{\mathrm{A}}$ is the Alfvén speed, defined with the magnetic field $B$ and the density $\rho$ as $v_{\mathrm{A}} \equiv B /(4 \pi \rho)^{1 / 2}$ (Balbus \& Hawley 1991). In protoplanetary disks, on the other hand, the ionization degree of material is low enough (e.g., Umebayashi \& Nakano 1988) that the effects 
of the ohmic dissipation and the ambipolar diffusion should be taken into account (e.g., Blaes \& Balbus 1994; Jin 1996; Gammie 1996; Sano et al. 2000). Here we examine the magnetorotationally unstable region in the disk with the temperature and density distributions obtained in $\S 3$ under the assumption that the ratio $c_{\mathrm{s} 0} / v_{\mathrm{A}}=10$ allover the disk. According to Sano et al. (2000), in which the stabilizing effect of the ohmic dissipation in protoplanetary disks is investigated, the disk is magnetorotationally unstable when

$$
2 \pi \max \left[v_{\mathrm{A}} / \Omega, \eta / v_{\mathrm{A}}\right] \leq H,
$$

where $\eta$ is the magnetic diffusivity. We here consider only the collisions between electrons and neutrals, mainly hydrogen molecules and helium atoms, as contributor to the diffusivity $\eta$ [the contributions of ions and charged grains are negligible at $x \geq 1 \mathrm{AU}$ (Sano et al. 2000)]. Thus $\eta$ is given by

$$
\eta \equiv c^{2} / 4 \pi \sigma_{c}=\left(c^{2} m_{e}<\sigma v>_{e} / 4 \pi e^{2}\right)\left(n / n_{e}\right),
$$

where $c, \sigma_{c}, m_{e}, e, n$, and $n_{e}$ are the light speed, the electrical conductivity, the electron mass and charge, the number densities of neutrals and electrons, respectively. For the momentum transfer rate between electrons and neutrals, $\langle\sigma v\rangle_{e}$, we use the experimental formula in Appendix of Sano et al. (2000; see also Hayashi 1981). Meanwhile, we evaluate the ionization degree $\xi \equiv n_{e} / n$ at $T \leq 10^{3} \mathrm{~K}$ with the ionization rate $\zeta$, the temperature $T$, and the molecular number density $n$ as $\xi=3.4 \times 10^{2} T^{1 / 4} \zeta^{1 / 2} n^{-1 / 2}$ (Gammie 1996), where $\zeta$ is represented as $\zeta=\zeta_{\mathrm{CR}} \exp \left(-\tau_{\mathrm{CR}}\right)+\zeta_{\mathrm{R}}$. Each of $\zeta_{\mathrm{CR}}$ and $\zeta_{\mathrm{R}}$ is the ionization rate by cosmic rays and radioactive elements, respectively. Following Umebayashi \& Nakano (1980), we adopt $\zeta_{\mathrm{CR}}=10^{-17} \mathrm{~s}^{-1}$ and $\zeta_{\mathrm{R}}=6.9 \times 10^{-23} \mathrm{~s}^{-1}$. The ionization depth by cosmic rays, $\tau_{\mathrm{CR}}$, is written as $\tau_{\mathrm{CR}}=\int_{z}^{\infty} \rho(x, z) d z / \chi_{\mathrm{CR}}$, where $\chi_{\mathrm{CR}}$ is the attenuation length and we use $\chi_{\mathrm{CR}}=96 \mathrm{~g} \mathrm{~cm}^{-2}$. At $T>10^{3} \mathrm{~K}$, where the thermal collision dominates the cosmic rays and the radioactive elements on ionization, we adopt the thermal ionization degree $\xi$ of Umebayashi (1983). In addition, in order to see the effect of ambipolar diffusion, we examine the ratio of the collision frequency between ions and neutrals to the epicyclic frequency, $\gamma_{\mathrm{d}} \rho_{\mathrm{i}} / \Omega$, where $\gamma_{\mathrm{d}} \approx 3.5 \times 10^{13} \mathrm{~cm}^{3} \mathrm{~g}^{-1} \mathrm{~s}^{-1}$ is the drag coefficient (e.g., Shu 1983) and $\rho_{\mathrm{i}}$ is the ion density (Blaes \& Balbus 1994; Hawley \& Stone 1998).

In Figure 4a we plot the resultant magnetorotationally unstable region (solid stripe) in the $x-z$ plane of the 'irradiated disk', whose density and temperature distributions are obtained from our two-dimensional numerical calculation. The figure shows that almost all the disk is magnetorotationally unstable except for the region near the equatorial plane of $2 \mathrm{AU}<r<10 \mathrm{AU}$. The ionization degree is high enough for the disk to be unstable in the inner region because of the thermal collisions, and in the surface and outer regions owing to the cosmic rays. Equation (17) suggests that the stable region spreads as $c_{\mathrm{s} 0} / v_{\mathrm{A}}$ becomes larger and vice versa (although we here examine only the case of $\left.c_{\mathrm{s} 0} / v_{\mathrm{A}}=10\right)$ since the relation $v_{\mathrm{A}} / \Omega<\eta / v_{\mathrm{A}}$ is satisfied allover the disk in this case (see also Sano et al. 2000). The stabilization due to the ambipolar diffusion is ignorable in this case $\left(\gamma_{\mathrm{d}} \rho_{\mathrm{i}} / \Omega \leq 10^{-2}\right.$ in allover the region which the ohmic dissipation dose not stabilize; see Stone \& Hawley 1998). For comparison we also plot the unstable region of the 'non-irradiated disk', which is obtained from the energy transport equation only in the vertical direction, in Figure $4 \mathrm{~b}$. 
The stable region in the 'irradiated disk' is smaller than that in the 'non-irradiated disk' because of its hotter temperature profile. We note that our assumption that the disk is heated by viscous dissipation at every radius $(\S 2.2)$ is conflict with the fact that the stable region exists in $2 \mathrm{AU}<r<$ 10AU. But our results will not be affected by this inconsistency since this region is heated not viscously but radiatively as we show in $§ 3$.

In summary we conclude that the turbulent source in a protoplanetary disk is not convective instability but magnetorotational instability under the conditions that the disk has the surface density distribution of minimum mass solar nebula and the viscous parameter of $\alpha=10^{-2}$.

\section{DUST SIZE DISTRIBUTION}

Protoplanetary disks are considered to consist at first of a few $\times 10^{-1} \mu \mathrm{m}$ sized dust grains, originating from the interstellar medium, which grow larger particles and at last lead to the planet formation through the processes of sticking, gravitational attraction, and gas accretion (e.g., Safronov 1969; Weidenschilling \& Cuzzi 1993; Beckwith, Henning \& Nakagawa 2000 and references therein). For the dust growth process numerous theoretical models have been proposed, for example, the collisional models in the laminar nebula and in the turbulent accretion disk, and the particle concentration model owing to turbulent motion (e.g., Kusaka, Nakano, \& Hayashi 1970; Weidenschilling 1980; Nakagawa, Nakazawa, \& Hayashi 1981; Mizuno 1989; Squires \& Eaton 1991; Klahr \& Henning 1997; Supulver \& Lin 2000). On the other hand, the evidences of dust grain growth in protoplanetary disks are observationally presented by means of the changes in their spectral energy distributions (e.g., Beckwith \& Sargent 1991; Beckwith et al. 2000; D'Alessio, Calvet, \& Hartmann 2001; Throop et al. 2001). In this section we discuss the dust size distribution in the disk, which has the density and temperature structures in $\S 3$ and the turbulent region obtained in $\S 4$ for the 'irradiated disk', dealing with the turbulent-eddy-trapping model of Klahr \& Henning (1997).

Dust particles can be trapped in a turbulent eddy in an accretion disk when the dust-gas friction time $\tau_{\mathrm{f}}$ satisfies the condition,

$$
\tau_{\mathrm{f}}<l \omega \min \left(1 / g_{z}, 1 / 2 \Omega \Delta V\right)
$$

where $l$ and $\omega$ are the size and the rotational frequency of the turbulent eddy, respectively, and $\Omega$ is the orbital frequency around the central star. $g_{z}=\Omega^{2} z$ is the vertical gravitational force and $\Omega \Delta V$ is the drag force caused by the difference in orbital velocity, $\Delta V$, between gaseous matter, undergoing the radial gas pressure, and the Keplerian rotating dust particles (e.g., Weidenschilling 1977). The dust-gas friction time $\tau_{\mathrm{f}}$ is given by

$$
\tau_{\mathrm{f}}=a_{\mathrm{p}} \rho_{\mathrm{d}} / c_{\mathrm{s}} \rho
$$

where $a_{\mathrm{p}}, \rho_{\mathrm{d}}, c_{\mathrm{s}}$, and $\rho$ are the radius and the density of dust particle, the thermal velocity and the density of gas, respectively. From equations (19) and (20) we can estimate the maximum size 
of dust grains, that can be trapped in turbulent eddies in the disk, as

$$
a_{\mathrm{p}, \max }=c_{\mathrm{s}} \rho z_{\infty} \Omega \min \left(1 / g_{z}, 1 / 2 \Omega \Delta V\right)
$$

where we take $l=z_{\infty}$ (disk height), $\omega=\Omega$, and $\rho_{\mathrm{d}}=1 \mathrm{~g} \mathrm{~cm}^{-3}$. In Figure 5 we plot the contour line of the maximum dust size distribution in the $x-z$ plane of the 'irradiated disk', which is estimated from equation (21) for the turbulent (magnetorotationally unstable) and dusty (with temperature of $T<2300 \mathrm{~K}$ ) region of the disk. The contour levels are $a_{\mathrm{p}, \max }=10^{-2}, 10^{-1}, 1,10$, and $10^{2} \mathrm{~cm}$ and the thick line displays $z=z_{\infty}$. The figure shows that dust particles can grow to be cm-size in the surface layer, which is because both of the temperature and the disk height are high enough there. These particles are expected to be under the influences of the outside activities, such as x-ray radiations, the FU Ori outbursts, and so on, which may lead to the chondrule and/or CAI formations (e.g., Jones et al. 2000). The dust size growth will also be connected with the opacity, the structure and the instabilities of the disk. We will consider these effects and the influence to the spectral energy distribution of the radiation from the disk in the next paper.

\section{SUMMARY}

In this paper we have evaluated the density and temperature structures of a protoplanetary disk with the surface density of the minimum mass solar nebula, rotating with the Keplerian velocity around a central star, which has the typical parameters of T Tauri stars, and under the influences of the heating processes via viscosity and the star. The so-called $\alpha$-model with $\alpha=10^{-2}$ is used here for turbulent viscosity. Assuming the vertical hydrostatic equilibrium and the local radiative equilibrium, and utilizing the numerical calculation of the two-dimensional radiative transfer equation, we have obtained the following conclusions:

1. The surface layer of the disk is heated and flared up via the irradiation from the inner hot disk rather than the central star in our disk model.

2. At the outer region, $x>1 \mathrm{AU}$, the radiative heating from the inner disk dominates the viscous heating because the temperature at the surface region is high and the disk is geometrically thick, owing to the irradiation from the inner hot region of the disk.

Making use of the resultant density and temperature profiles, we have examined the convective and the magnetorotational instabilities of the disk, which are expected to induce the turbulent motion, and then, bring about the angular momentum and mass transfer, and/or the energy transport. As a result, we have found that:

3. The whole disk is convectively stable since the dusty region is not heated by the viscous dissipation from the midplane but the radiative heating from the inner disk.

4. Almost all of the disk is magnetorotationally unstable as the ionization degree is high enough that the stabilizations by ambipolar diffusion and ohmic dissipation are negligible, except 
for the region near the midplane of $2 \mathrm{AU}<x<10 \mathrm{AU}$.

Turbulent eddies, excited by magnetorotational instability, will trap dust grains to facilitate their growth under some conditions. We have discussed the size distribution of dust particles in the disk, which suggests that:

5. The dust particles can grow to be cm-size in the surface layer region because of the high temperature distribution and the large disk height. These particles are expected to be exposed to the outside activities, such as x-ray radiations, the FU Ori outbursts, and so on, which may affect the dust grain evolutions.

The influences of the evolution of turbulent motion, the dust size distribution, and the surface density distribution on the structure, the instabilities, and the spectral energy distribution of the disk should be studied in the next work.

I am grateful to the referee, Dr. I. Hubeny, for his valuable comments, which improved the clarity of our discussions. I would like to thank Dr. T. Nakamoto, Dr. T. Sano, and Dr. E.I. Chiang for very useful comments. I am also appreciative to Dr. S. Inutsuka, Dr. S. Mineshige, and Dr. H. Kamaya for continuous encouragement. This work is financially supported by Research Fellowships of the Japan Society for the Promotion of Science for Young Scientists, 03055.

\section{A. Numerical Method}

\section{A.1. Iterative Method to Calculate Temperature and Density Profiles}

At first we note how we evaluate iteratively the temperature and density distributions. In the following $T^{i}(r, \Theta)$ and $\rho^{i}(x, z)$ represent the results of the $i$-th iterative calculation of the processes (II)-(V), while $T^{j}(r, \Theta)$ is the result of the $j$-th iteration of (a)-(f). The iterative methods are as follows:

(I) Initially put the density and temperature profiles of optically thick accretion disk, $\rho^{0}\left({ }^{\forall} x,{ }^{\forall} z\right)$ and $T^{0}\left({ }^{\forall} r,{ }^{\forall} \Theta\right)$. They are derived in the conventional manner from the hydrostatic equation (1) in $\S 2.1$ and the energy transfer equation in the vertical direction via diffusion (e.g., Lin \& Papaloizou 1980, 1985),

$$
\frac{4 c}{3 \kappa_{\mathrm{R}} \rho} \frac{d\left(a T^{4}\right)}{d z}=\sigma T_{\mathrm{eff}}^{4},
$$

where $a$ is the radiative constant, defined with the Stefine-Boltzmann constant $\sigma$ and the light speed $c$ as $a=4 \sigma / c$. In order to solve equation (A1) we use a condition (9/4) $\Sigma \alpha c_{\mathrm{s}_{0}}^{2} \Omega=\sigma T_{\text {eff }}^{4}$, where $c_{\mathrm{s} 0}^{2}=k T(x, z=0) / m_{\mu}$ and $T_{\text {eff }}=T\left(x, z=z_{\mathrm{p}}\right) . \quad z_{\mathrm{p}}$ is the photospheric disk height where the optical depth from the disk surface $z_{\infty}$ becomes $2 / 3$, that is, $\tau_{z}=\int_{z_{\infty}}^{z_{\mathrm{p}}} \kappa_{\mathrm{R}} \rho d z=2 / 3$. Here $\kappa_{\mathrm{R}}$ is the Rosseland mean opacity described in $\S 2.4$. The condition means the energy balance in the vertical direction between the viscous heating at the midplane and the blackbody radiative 
cooling at $z=z_{\mathrm{p}}$ (e.g., Meyer \& Meyer-Hofmeister 1982). These initial density and temperature distributions are plotted and used in $\S 3$ and $\S 4$ as 'non-irradiated disk' (which means that only vertical energy transport is took into account), and compared with the 'irradiated disk' (obtained from our two-dimensional radiative transfer calculation below).

(II) Evaluate the temperature distribution $T^{i}\left({ }^{\forall} r,{ }^{\forall} \Theta\right)$ by using $\rho^{i-1}\left({ }^{\forall} x,{ }^{\forall} z\right)$ and iteratively solving equations (7)-(14) as we mentioned in $\S 2.3$ : (a) Calculate the intensity at each point $I_{{ }_{\nu}}\left({ }^{\forall} r,{ }^{\forall} \Theta ;{ }^{\forall} \mu,{ }^{\forall} \phi\right)$ by using tentative temperature distribution $T^{j}\left({ }^{\forall} r,{ }^{\forall} \Theta\right)$ and integrating equation (13) with the Short Characteristics method described in $\S$ A.2. (b) Substitute the intensity at a point $I_{\nu}\left(r, \Theta ;{ }^{\forall} \mu,{ }^{\forall} \phi\right)$ into equation (8) to obtain the mean intensity $J_{\nu}(r, \Theta)$ at that point. (c) Evaluate the corrected temperature $T^{* j}(r, \Theta)$ at that point from equation of radiative equilibrium (7). (d) Estimate the temperature correction in context of the Newton-Raphson method (e.g., Press et al. 1985) as

$$
\delta T^{j}(r, \Theta)=\frac{\Delta_{T}^{j}}{\left(\partial \Delta_{T} / \partial T\right)^{j}}=\frac{\Delta_{T}^{j}}{\left(\Delta_{T}^{j-1}-\Delta_{T}^{j}\right) / \delta T^{j-1}}
$$

for $\Delta_{T}^{j}(r, \Theta) \geq 0.1$ and $\delta T^{j}(r, \Theta)=0$ for $\Delta_{T}^{j}(r, \Theta)<0.1$, where $\Delta_{T}^{j}(r, \Theta) \equiv\left\{T^{* j}(r, \Theta)-\right.$ $\left.T^{j}(r, \Theta)\right\} / T^{j}(r, \Theta)$. (e) Compute $\delta T^{j}\left({ }^{\forall} r,{ }^{\forall} \Theta\right)$ for all grid points in the same manner. (f) Return to step (a) with the new tentative temperature $T^{j+1}(r, \Theta)=T^{j}(r, \Theta)+\delta T^{j}(r, \Theta)$ at each point, and repeat these operations until $\left|\Delta_{T}^{j}\left({ }^{\forall} r,{ }^{\forall} \Theta\right)\right|<0.1$ and $\left|T^{j}(r, \Theta)-T^{j-1}(r, \Theta)\right| / T^{j-1}(r, \Theta)<0.1$ for $\left({ }^{\forall} r,{ }^{\forall} \Theta\right)$.

(III) Making use of $T^{i}\left({ }^{\forall} r,{ }^{\forall} \Theta\right)$, compute the density distribution $\rho^{i}\left({ }^{\forall} x,{ }^{\forall} z\right)$ from equation (1) as we stated in $\S 2.1$.

(IV) Estimate $T^{i+1}(r, \Theta)$ in the optically thick region by using $\rho^{i}\left({ }^{\forall} x,{ }^{\forall} z\right)$ and solving equation (A1) as we stated in the process (I). This is because we can hardly get the adequate temperature from a wrong initial value of $T^{j=1}(r, \Theta)$ in very optically thick region, owing to the approximate treatment of integral (13) and to the determination of the temperature correction $\delta T^{j}(r, \Theta)$ in (II). As we describe in $\S$ A.2, the Short Characteristics method to integrate equation (13) lets $I_{\nu}(r, \Theta ; \mu, \phi) \rightarrow B_{\nu}[T(r, \Theta)]$, which leads to $\Delta_{T}^{j}(r, \Theta) \rightarrow 0$ and then $\delta T^{j}(r, \Theta) \rightarrow 0$, for very optically thick limit. (This is also why we choose the initial density and temperature distributions as $[\mathrm{I}]$.

(V) Return to step (II) until $\left|\rho^{i}(x, z)-\rho^{i-1}(x, z)\right| / \rho^{i-1}(x, z)<0.1$ and $\left|T^{i}(r, \Theta)-T^{i-1}(r, \Theta)\right| / T^{i-1}(r, \Theta)<$ 0.1 for $\left({ }^{\forall} x,{ }^{\forall} z\right)$ and $\left({ }^{\forall},{ }^{\forall} \Theta\right)$.

\section{A.2. Short Characteristics Method to Calculate Intensity}

Next we mention the numerical method to integrate equation (13) in order to get the specific intensity $I_{\nu}(r, \Theta ; \mu, \phi)$. Since it costs vast CPU time to compute the integration from $s^{\prime}=0$ to $s$ in all directions $(\mu, \phi)$ and all frequencies $\nu$, we here adopt an efficient and convenient algorithm, 
the Short Characteristics method in the spherical coordinates (Dullemond \& Turolla 2000; see also Mihalas, Auer, \& Mihalas 1978, Olson \& Kunasz 1987, Auer \& Paletou 1994). Following them, we approximate to calculate the intensity at a point $P$ under the assumption of neglecting the scattering (that is, only the black body radiation $B_{\nu}[T(r, \Theta)]$ is considered for source function at each point) as

$$
I_{\nu}(P ; \mu, \phi) \approx e^{-\tau_{\nu}} I_{\nu}(U ; \mu, \phi)+u_{\nu} B_{\nu}(U)+p_{\nu} B_{\nu}(P)+d_{\nu} B_{\nu}(D),
$$

where $U$ and $D$ represent the upstream and downstream points along a ray directed to $(\mu, \phi)$, and $\tau_{\nu}$ is the optical depth between the points $U$ and $P$. The coefficients $u_{\nu}, p_{\nu}$, and $d_{\nu}$ are functions of the optical depths from $U$ to $P$ and from $P$ to $D$ (see e.g., Dullemond \& Turolla 2000 for detailed determination of the coefficients). The intensity (A2) approaches $I_{\nu}(P ; \mu, \phi) \rightarrow I_{\nu}(U ; \mu, \phi)$ for optically thin limit, $\tau_{\nu} \rightarrow 0$, and $I_{\nu}(P ; \mu, \phi) \rightarrow B_{\nu}(P)$ for optically thick limit, $\tau_{\nu} \rightarrow \infty$. Making use of this method, we can obtain $I_{{ }_{\nu}}\left({ }^{\forall} r,{ }^{\forall} \Theta ;{ }^{\forall} \mu,{ }^{\forall} \phi\right)$ systematically from the intensity at boundaries (see below) and the temperature at each point $T\left({ }^{\forall} r,{ }^{\forall} \Theta\right)$.

In this calculation we use the spatial grid of $\left(r_{k}, \Theta_{l}\right)$, where $k=1,40$ and $l=1,80$. In order to resolve the inner region of the disk, the radial grid is put logarithmically as $r_{k+1}=p r_{k}$, where $p$ is a constant, determined as $r_{1}=r_{\text {in }}$ and $r_{40}=r_{\text {out }}$ are satisfied. We assign the inner radius as $r_{\text {in }}=R_{*}=2 R_{\odot}$ and the outer radius as $r_{\text {out }}=100 \mathrm{AU}$. The azimuthal grid points are equally spaced in $0<\Theta<\pi / 2$. For the direction of the ray-path $(\mu, \phi)$, we put 32 points at the maximum for $\mu$ in $0 \leq \mu \leq 1$ (see Dullemond \& Turolla 2000 in detail for choosing the $\mu$-grid), and equally spaced 8 points for $\phi$ in $0<\phi<\pi / 2$. The frequency is divided logarithmically into 32 points in $10^{10} \leq \nu \leq 10^{15} \mathrm{~Hz}$. The boundary conditions that we adopt here are as follows: $I_{\nu}\left(r=r_{1}, \Theta ; \mu>0, \phi\right)=B_{\nu}\left(T=T_{*}=4000 \mathrm{~K}\right)$ is taken for the intensity to the outward direction at the inner radius $r=r_{1}$, and $I_{\nu}\left(r=r_{40}, \Theta ; \mu<0, \phi\right)=B_{\nu}(T=10 \mathrm{~K})$ for the inward intensity at the outer radius $r=r_{40}$ (the gaseous matter in molecular clouds is considered to have the equilibrium temperature of about $T=10 \mathrm{~K}$ between heating by cosmic rays and cooling by molecular line emissions; e.g., Myers 1978). At $\Theta=\Theta_{1}$ we take the symmetric condition against $z$-axis $(\Theta=0)$, and at $\Theta=\pi / 2$ we put $I_{\nu}(r, \Theta=\pi / 2 ; \mu, \phi)=B_{\nu}\left(T=T_{\text {vis }}\right)$. We note that the boundary conditions at $r=R_{*}$ and $\Theta=\pi / 2$ reproduce the heating sources, the central star and the viscous dissipation at the midplane, respectively, that we described in $\S 2.2$ and $\S 2.3$.

\section{REFERENCES}

Adams, F.C. \& Shu, F.H. 1985, ApJ, 296, 655

Adams, F.C. \& Shu, F.H. 1986, ApJ, 308, 836

Adams, F.C., Lada, C.J., \& Shu, F.H. 1987, ApJ, 312, 788

Alexander, D.R. \& Ferguson, J.W. 1994, ApJ, 437, 879 
Auer, L.H., \& Paletou, F. 1994, A\&A, 285, 675

Balbus, S.A., \& Hawley, J.F. 1991, ApJ, 376, 214

Balbus, S.A., \& Hawley, J.F. 1998, Rev. Mod. Phys., 70, 1

Bally, J., O’Dell, C.R., \& McCaurean, M.J. 2000

Beckwith, S.V.W., Henning, T., \& Nakagawa, Y. 2000 in Protostars and Planets IV, ed. V.G. Mannings, A.P. Boss, \& S.S. Russell (Tuscon: Univ. Arizona Press), 533

Beckwith, S.V.W., \& Sargent, A.I. 1993, in Protostars and Planets III, ed. E.H. Levy \& J. Lunine (Tuscon, Univ. Arizona Press), 521

Bell, K.B. 1999, ApJ, 526, 411

Bell, K.R., \& Lin, D.N.C. 1994, ApJ, 427, 987

Blaes, O.M., \& Balbus, S.A. 1994, ApJ, 421, 163

Bodenheimer, P. 1995, ARA\&A, 33, 199

Burrows, C. J., et al. 1996, ApJ, 473, 437

Cabot, W., Canuto, V.M., Hubuckyj, O., \& Pollack, J.B. 1987, Icarus, 69, 423

Cameron, A.G.W. 1978, Moon Planets, 18, 5

Cameron, A.G.W., \& Pine, M.R. 1973, Icarus, 18, 377

Chiang, E.I. \& Goldreich, P. 1997, ApJ, 490, 368

Cohen, M. 1974, MNRAS, 169, 257

Cox, J.P. 1980, Theory of Stellar Pulsation (Princeton: Princeton University Press)

D’Alessio, P., Calvet, N., Hartmann, L. 2001, ApJ, 553, 321

D’Alessio, P., Cantó, J., Calvet, N., \& Lizano, S. 1998, 500, 411

Draine, B., \& Lee, H.M. 1984, ApJ, 285, 89

Dullemond, C. P. \& Turolla, R. 2000, A\&A, 360, 1187

Efstathiou, A. \& Rowan-Robinson, M. 1990, MNRAS, 245, 275

Elmegreen, B.G. 1978, Moon Planets, 19, 261

Gammie, C.F. 1996, ApJ, 457, 355 
Haisch, K.E., Lada, E.A., \& Lada, C.J. 2001, ApJ, 553, L153

Hartmann, L. 1998, Accretion processes in star formation (New York: Cambridge University Press)

Hawley, J.F. \& Stone, J.M. 1998, ApJ, 501, 758

Hayashi, C., Nakazawa, K., \& Nakagawa, Y. 1985, in Protostars and Planets II, ed. D.C. Black \& M.S. Mathews (Tuscon: Univ. Arizona Press), 1100

Hayashi, M. 1981, Inst. Plasma Phys. Japan Int. Rep. IPPJ-AM-19

Hubeny, I. 1990, ApJ, 351, 632

Hubeny, I. 1991, in IAU Colloq. 129, Structure and Emission Properties of Accretion Disks, ed. C. Bertout et al (Gif sur Yvette: Editions frontieres), 227

Jin, L. 1996, ApJ, 457, 798

Jones, R.H., Lee, T., Connolly, H.C., Jr., Love, S.G., \& Shang, H. 2000, Protostars and Planets IV, ed. V.G. Mannings, A.P. Boss, \& S.S. Russell (Tuscon: Univ. Arizona Press), 927

Kato, S., Fukue, J., \& Mineshige, S. 1998, Black-hole Accretion Disks (Kyoto: Kyoto Univ. Press)

Kawazoe, E., \& Mineshige, S. 1993, PASJ, 45, 715

Kenyon, S. J., \& Hartmann, L. 1987, ApJ, 323, 714

Kenyon, S. J., \& Hartmann, L. 1995, ApJS, 101, 117

Klahr, H.H., \& Henning, T. 1997, Icarus, 128, 213

Kley, W., Papaloizou, J.,C.B., \& Lin, D.N.C. 1993, ApJ, 416, 679

Kusaka, T., Nakano, T., \& Hayashi, C. 1970, Prog. Theor. Phys., 44, 1580

Lin, D.N.C., \& Papaloizou, J.C.B. 1980, MNRAS, 191, 37

Lin, D.N.C., \& Papaloizou, J.C.B. 1985, in Protostars and Planets II, ed. D.C. Black \& M.S. Mathews (Tuscon: Univ. Arizona Press), 981

Lynden-Bell, D., \& Pringle, J.E. 1974, MNRAS, 168, 603

Meyer, F. \& Meyer-Hofmeister, D. 1982, A\&A, 106, 34

Mihalas, D.M. 1978, Stellar Atmospheres (San Francisco: Freeman)

Mihalas, D.M., Auer, L.H., \& Mihalas, B.R. 1978, ApJ, 220, 1001

Mizuno, H. 1989, Icarus, 80, 189 
Myers, P.C. 1978, ApJ, 225, 380

Nakagawa, Y., Nakazawa, K., \& Hayashi, C. 1981, Icarus, 45, 517

Nomura, H., \& Mineshige, S. 2000, ApJ, 536, 429

O'Dell, C.R. 1998, AJ, 115, 263

O’Dell, C.R., Valk, J.H., Wen, Z., \& Meyer, D.M. 1993, ApJ, 403, 678

Olson, G. \& Kunasz, P. 1987, JQSRT, 38, 325

Padgett, D.L., Brandner, W., Stapelfeldt, K.R., Strom, S.E., Terebey, S., Koerner, D. 1999, AJ, 117,1490

Papaloizou, J.C.B., \& Lin, D.N.C. 1995, ARA\&A, 33, 505

Press, W.H., Flannery, B.P., Teukolsky, S.A., \& Vetterling, W.T. 1985, Numerical Recipes (Cambridge: Cambridge Univ. Press)

Pringle, J.E. 1981, ARA\&A, 19, 137

Ruden, S.P., Papaloizou, J.C.B., \& Lin, D.N.C. 1988, ApJ, 329, 739

Safronov, V.S. 1969, Evolution of the Protoplanetary Cloud and Formation of the Earth and Planets (Moscow: Nauka Press)

Sano, T., Miyama, S.M., Umebayashi, T., \& Nakano, T. 2000, ApJ, 543, 486

Sekiya, M. 2000, EPS, 52, 517

Shakura, N.I., \& Sunyaev, R.A., 1973, A\&A, 24, 337

Shu, F.C. 1983, ApJ 273, 202

Shu, F.H., Adams, F.C., \& Lizano, S. 1987, ARA\&A, 25, 23

Spitzer, L., Jr. 1978, Physical Processes in the Interstellar Medium (New York: Wiley)

Squires, K.D., \& Eaton, J.K. 1991, Phys. Fluids A, 3, 1169

Stauffer, J. R., Prosser, C. F., Hartmann, L., \& McCaughrean, M. J. 1994, AJ, 108, 1375

Stone, J.M., Gammie, C.F., Balbus, S.A., \& Hawley, J.F. 2000, in Protostars and Planets IV, ed. V.G. Mannings, A.P. Boss, \& S.S. Russell (Tuscon: Univ. Arizona Press), 589

Stone, J.M., \& Balbus, S.A. 1996, 464, 364

Supulver, K.D. \& Lin, D.N.C. 2000, Icarus, 146, 525 
Troop, H.B., Bally, J., Esposito, L.W., \& McCaughrean, M.J. 2001, Science, 292, 1686

Umebayashi, T. 1983, Prog. Theor. Phys., 69, 480

Umebayashi, T., \& Nakano, T. 1980, PASJ, 32, 405

Umebayashi, T., \& Nakano, T. 1988, Prog. Theor. Phys. Suppl., 96, 151

Velhikov, E.P. 1959, J. Exp. Theor. Phys., 36, 1398

Weidenschilling, S.J. 1977, MNRAS, 180, 57

Weidenschilling, S.J. 1980, Icarus, 44, 172

Weidenschilling, S.J. \& Cuzzi, J.N. 1993, in Protostars and Planets III, ed. E.H. Levy \& J. Lunine (Tuscon, Univ. Arizona Press), 1031

Wilking, B. A., Lada, C. J., \& Young, E. T. 1989, ApJ, 340, 823

Zel'dovich, YaB. 1981, Proc. R. Soc. Lond., A, 374, 299 

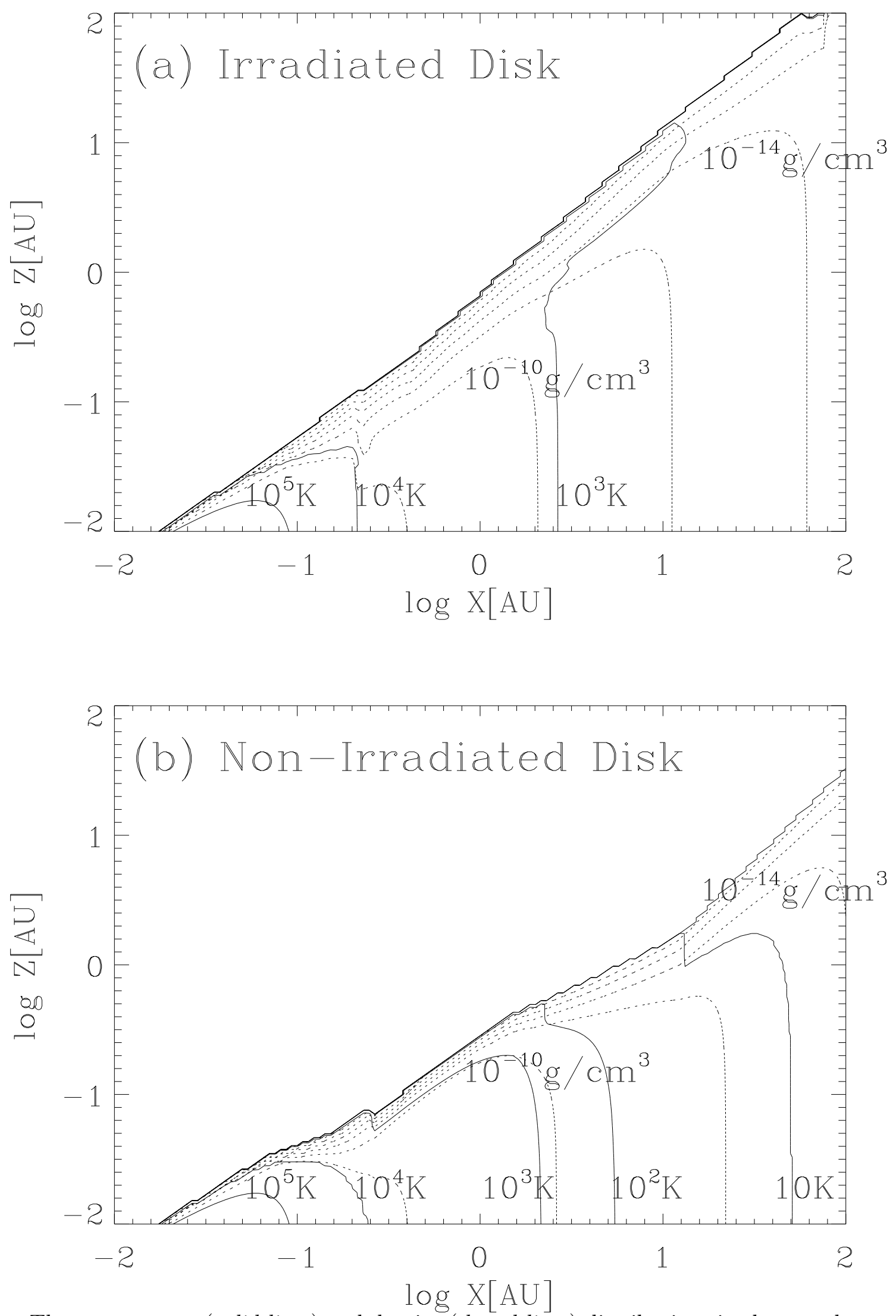

Fig. 1. - The temperature (solid lines) and density (dotted lines) distributions in the $x-z$ plane of (a) 'irradiated disk' (obtained from 2-D radiative transfer calculation) and (b) 'non-irradiated disk' (derived from only vertical energy transfer equation). The contour levels are $T=10,10^{2}, 10^{3}, 10^{4}$, and $10^{5} \mathrm{~K}$ for the temperature, and $\rho=10^{-18}, 10^{-16}, 10^{-14}, 10^{-12}, 10^{-10}$, and $10^{-8} \mathrm{~g} \mathrm{~cm}^{-3}$ for the 

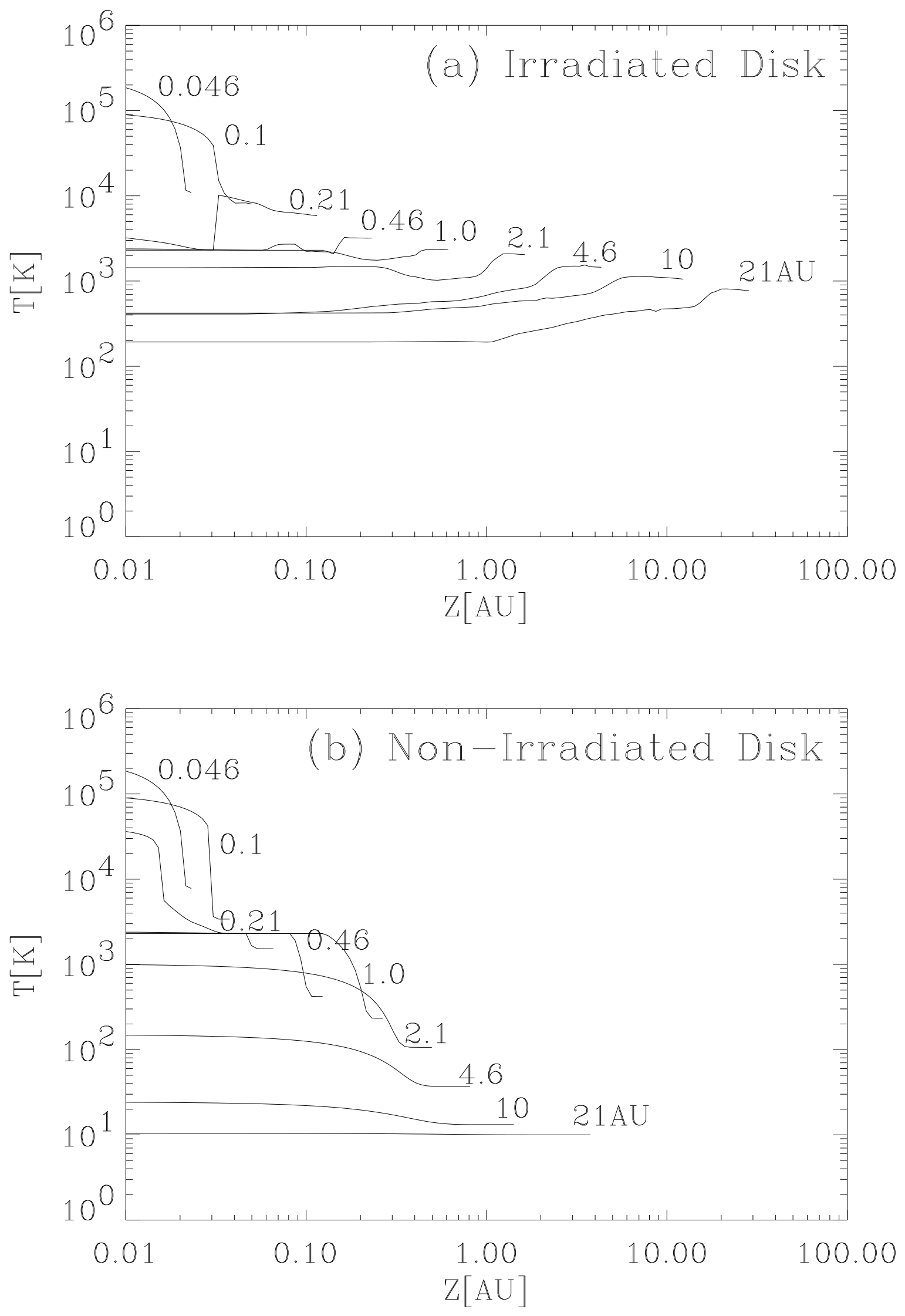

Fig. 2.- The vertical temperature profiles at $x=0.046,0.1,0.21,0.46,1.0,2.1,4.6,10$, and $21 \mathrm{AU}$ of (a) 'irradiated disk' and (b) 'non-irradiated disk'. 

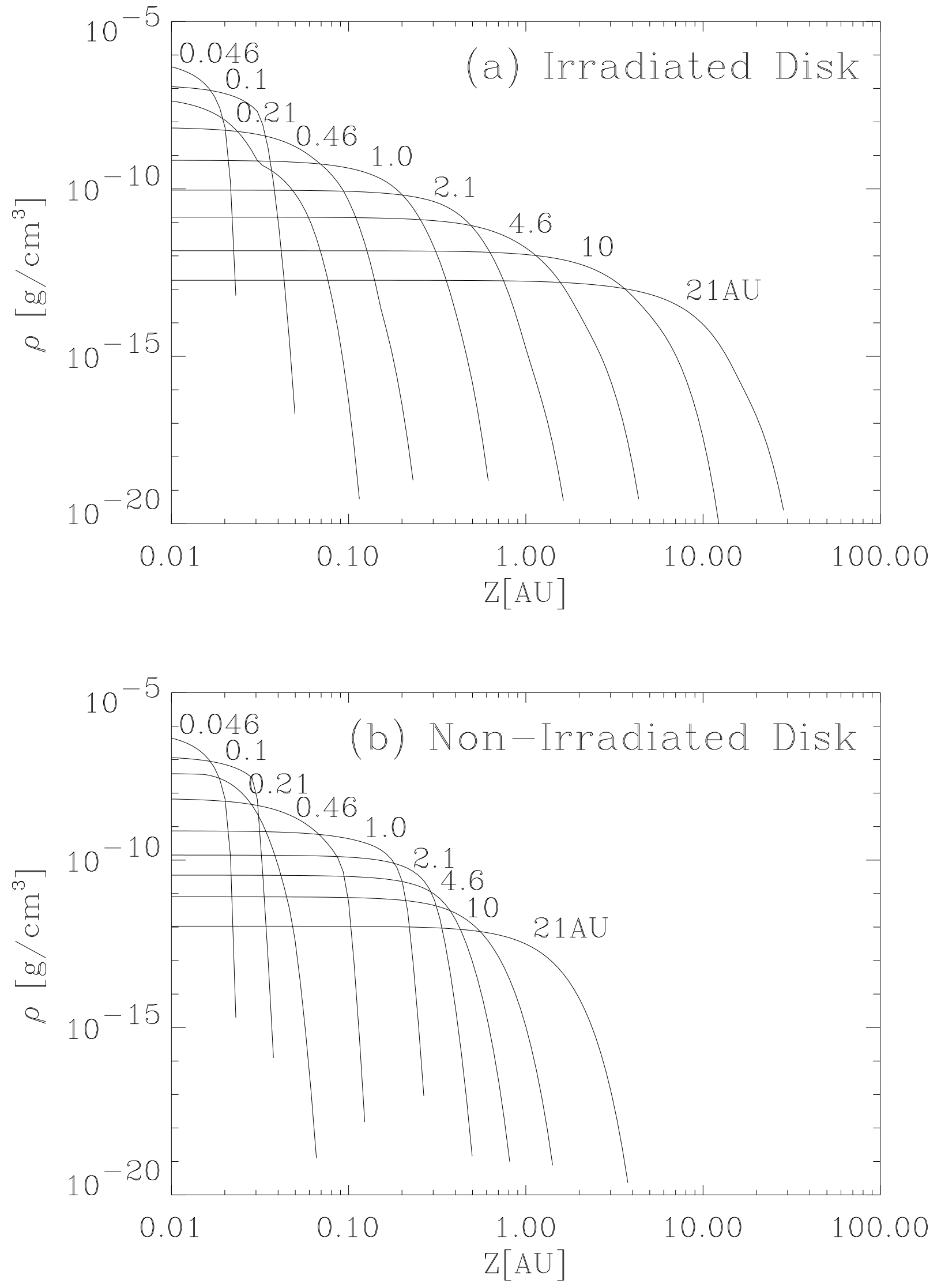

Fig. 3. - The same as Figure 2 but the vertical density profiles. 

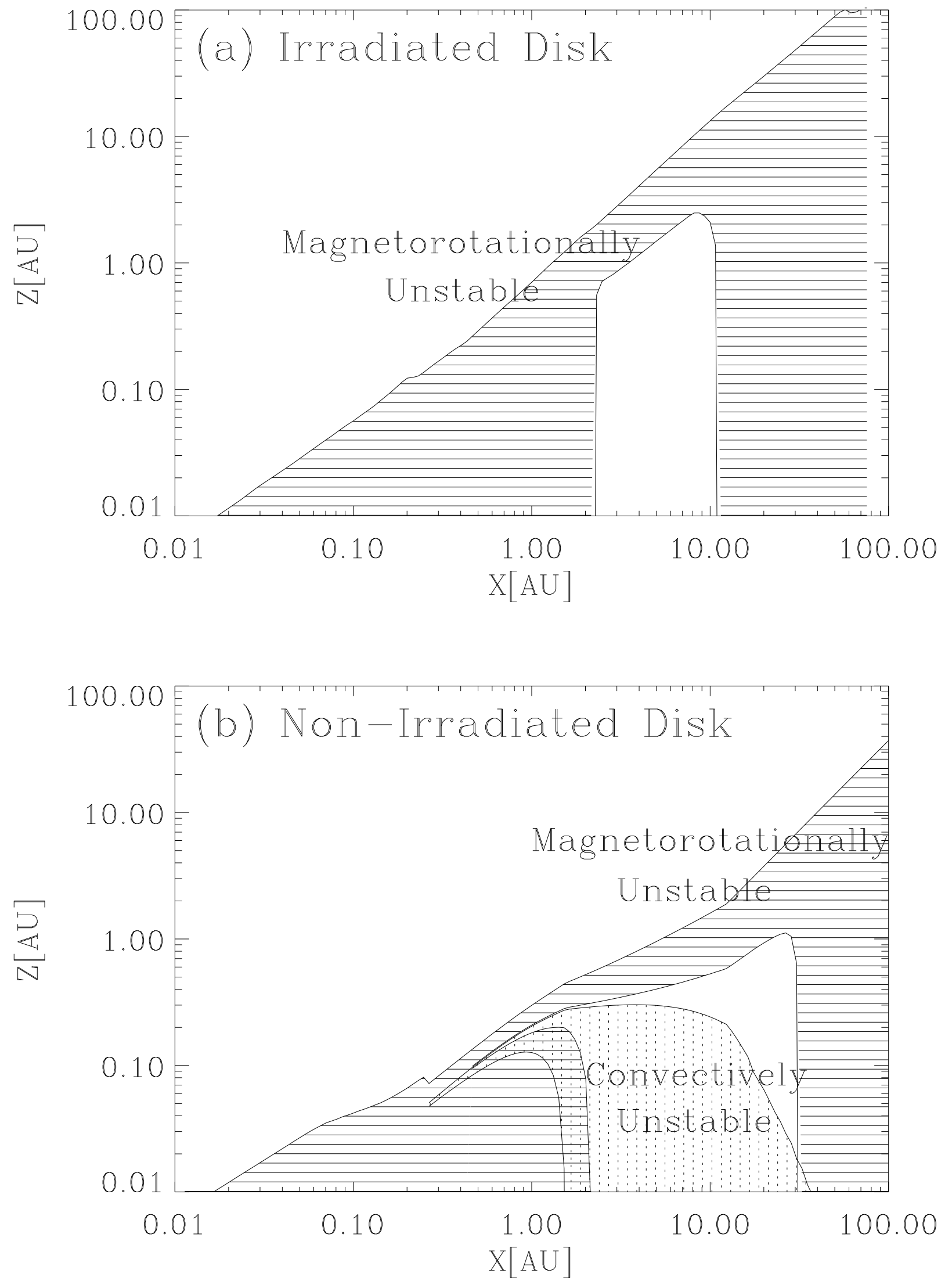

Fig. 4.- The convectively (dotted stripe) and the magnetorotationally (solid stripe) unstable regions in the $x-z$ plane of (a) 'irradiated disk' and (b) 'non-irradiated disk'. The whole 'irradiated disk' 


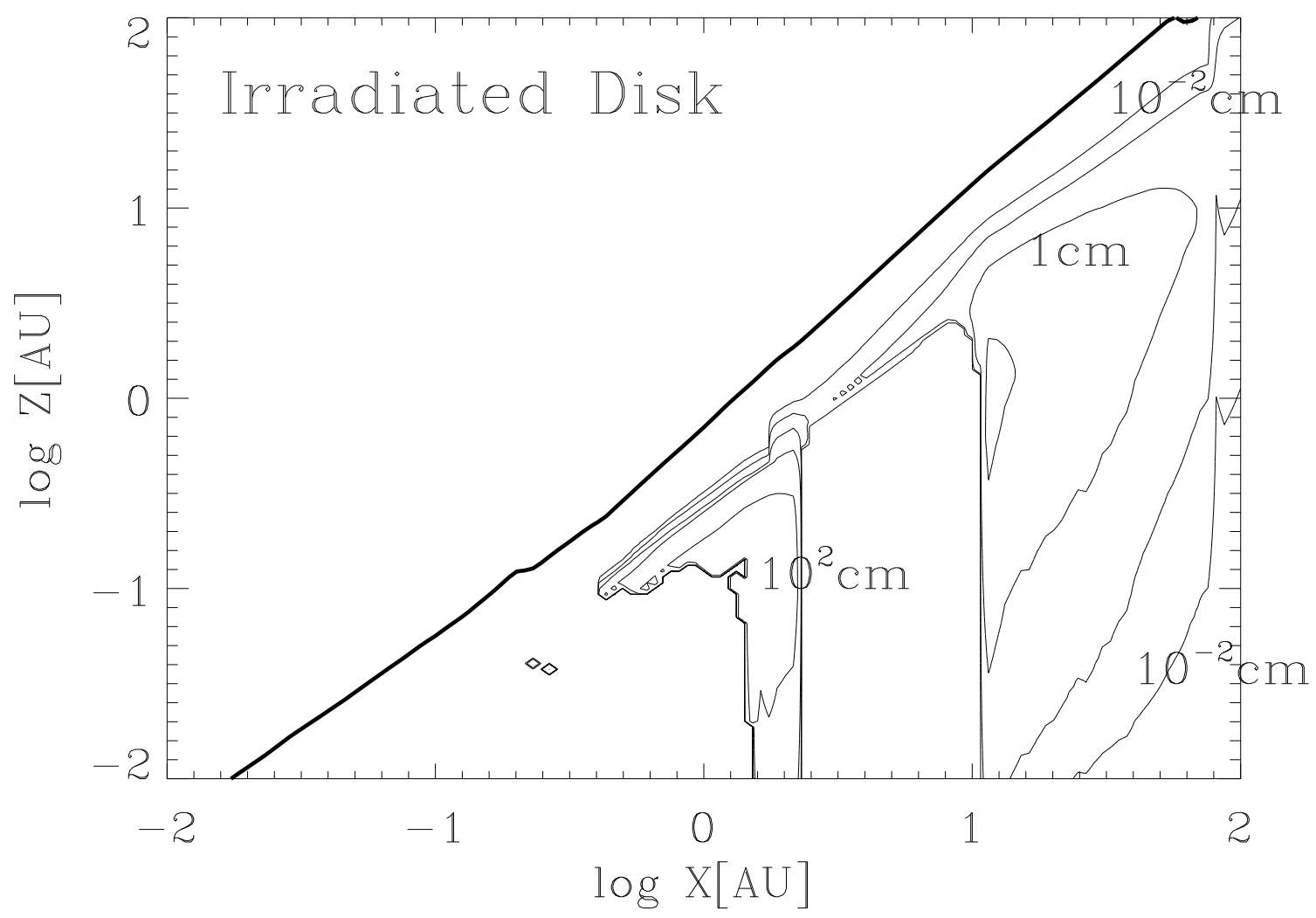

Fig. 5.- The maximum dust size distribution in the $x-z$ plane of 'irradiated disk'. The contour levels are $a_{\mathrm{p}, \max }=10^{-2}, 10^{-1}, 1,10$, and $10^{2} \mathrm{~cm}$. The thick line displays $z=z_{\infty}$. The dust particles can grow to be cm-size in the surface layer, which are expected to be under the influences of the outside activities. 